\title{
LARGE TIME STABILITY OF THE MAXWELL STATES*
}

\author{
FUMIOKI ASAKURA ${ }^{\dagger}$
}

\author{
Dedicated to Professor Kiyoshi Mochizuki on his sixtieth birthday
}

\begin{abstract}
We study the Cauchy problem for a $2 \times 2$-system of conservation laws: $v_{t}-u_{x}=0$, $u_{t}-\sigma(v)_{x}=0$ describing the phase transition. Two constant states satisfying the Maxwell equalarea principle constitute an admissible stationary solution; a small perturbation of these Maxwell states will be our initial data. We shall show that: there exists a global in time propagating phase boundary which is admissible in the sense that it satisfies the Abeyaratne-Knowles kinetic condition; the states outside the phase boundary tend to the Maxwell states as time goes to infinity.
\end{abstract}

1. Introduction. The van der Waals equation of state:

$$
p(v)=\frac{R T}{v-b}-\frac{a}{v^{2}}, \quad v>b
$$

provides a simple analytical relation between the pressure and the specific volume and describes in a satisfactory manner the behavior of many substances over a wide range of temperatures and pressures. This equation of state represents homogeneous isothermal liquid-vapor states, but does not agree with the requirements of stable inhomogeneous state composed of a liquid and a vapor part. We know that this inhomogeneous state is in thermal equilibrium at the saturation pressure if and only if the pressure satisfies the Maxwell equal-area principle; the liquid and the vapor state determined by this principle are called the Maxwell states. The dynamic changes of isothermal liquid-vapor states are described by the equations of gas dynamics with the van der Waals equation of state. Here the above Maxwell states constitute a weak stationary solution. Now a simple question arises: if these Maxwell states are compactly perturbed, then, as time goes on, the states come back or not to the Maxwell states. In this paper, we shall find one way to say "Yes".

We study a $2 \times 2$-system of conservation laws:

$$
v_{t}-u_{x}=0, \quad u_{t}-\sigma(v)_{x}=0 .
$$

Here $\sigma(v)$ is a $C^{2}$ - function and there exist $\alpha, \beta(\alpha<\beta)$ such that

$$
\sigma^{\prime}(v)= \begin{cases}>0 & \text { for } v<\alpha \\ <0 & \text { for } \alpha<v<\beta \\ >0 & \text { for } v>\beta\end{cases}
$$

and we further assume that

$$
\sigma^{\prime \prime}(v) \neq 0, \quad \text { for } \quad v<\alpha, v>\beta .
$$

The system of equations is hyperbolic for $v<\alpha, v>\beta$ and elliptic for $\alpha<v<\beta$; the region $\Omega_{\alpha}=\{(v, u) ; v<\alpha\}$ is called the $\alpha$-phase and $\Omega_{\beta}=\{(v, u) ; v>\beta\}$ the $\beta$-phase. We consider the initial data of the form:

$$
(v(x, 0), u(x, 0))= \begin{cases}\left(v_{L}(x), u_{L}(x)\right) \in \Omega_{\alpha} & \text { for } x<0 \\ \left(v_{R}(x), u_{R}(x)\right) \in \Omega_{\beta} & \text { for } x>0 .\end{cases}
$$

\footnotetext{
${ }^{*}$ received April 15, 1998; revised February 23, 1999.

${ }^{\dagger}$ Faculty of Engineering, Osaka Electro-Communication University, Japan (asakura@isc.osakac. ac.jp).
} 
If the initial data $U_{R}(x)=\left(v_{R}(x), u_{R}(x)\right), U_{L}(x)=\left(v_{L}(x), u_{L}(x)\right)$ are constant states $U_{R}, U_{L}$, the initial value problem is called the Riemann problem. The jump discontinuity of the form:

$$
(v(x, t), u(x, t))= \begin{cases}\left(v_{-}, u_{-}\right) & \text {for } x<s t \\ \left(v_{+}, u_{+}\right) & \text {for } x>s t\end{cases}
$$

( $v_{ \pm}, u_{ \pm}$: constants) is said to be a phase boundary if the states belong to the different phases: $U_{-}=\left(v_{-}, u_{-}\right) \in \Omega_{\alpha}, U_{+}=\left(v_{+}, u_{+}\right) \in \Omega_{\beta}$ and satisfy the Rankine-Hugoniot condition:

$$
\left\{\begin{array}{l}
s\left(u_{+}-u_{-}\right)=-\left(\sigma\left(v_{+}\right)-\sigma\left(v_{-}\right)\right), \\
s\left(v_{+}-v_{-}\right)=-\left(u_{+}-u_{-}\right) .
\end{array}\right.
$$

Sometimes it will be convenient to use the term phase boundary to refer the phase front $x=s t$. Clearly, two states satisfying

$$
\sigma\left(v_{+}\right)=\sigma\left(v_{-}\right), \quad u_{+}=u_{-}
$$

constitute a stationary $(s=0)$ phase boundary. On the other hand, a propagating phase boundary is such that the propagation speed $s$ is non-zero (see James [15]). There exists a unique pair $\left(v_{m}, v_{m}^{*}\right)$ satisfying $v_{m}<\alpha, v_{m}^{*}>\beta$ and

$$
\int_{v_{m}}^{v_{m}^{*}} \sigma(v) d v=\sigma\left(v_{m}\right)\left(v_{m}^{*}-v_{m}\right), \quad \sigma\left(v_{m}^{*}\right)=\sigma\left(v_{m}\right) .
$$

By choosing any $u_{m}=u_{m}^{*} \in R$, states, denoted in particular by

$$
U_{m}=\left(v_{m}, u_{m}\right), \quad U_{m}^{*}=\left(v_{m}^{*}, u_{m}^{*}\right),
$$

are called the Maxwell states and constitute an important stationary phase boundary. The Maxwell states are admissible in the sense that they minimize the entropy rate for $v$ close to $v_{m}$ and stationary phase boundaries are not admissible unless $v_{-}=$ $v_{m}$ (Hattori's theorem [13], see also Dafermos [9])). This admissibility condition is generalized by Abeyaratne and Knowles [1], [2] in ingenious way which we review briefly in the following.

The system of conservation laws (1.1) is endowed with the canonical entropy pair (total mechanical energy [17]):

$$
\eta(U)=\frac{1}{2} u^{2}+\int_{0}^{v} \sigma(w) d w, \quad q(U)=-u \sigma(v) .
$$

Let the interval $\left[x_{1}, x_{2}\right]$ contain a finite number of jump discontinuities (1.5). The local entropy is given by

$$
H\left(t ; x_{1}, x_{2}\right)=\int_{x_{1}}^{x_{2}} \eta(U(x, t)) d x .
$$

By direct computation, we find that the rate of decay of local entropy is expressed as

$$
\frac{d H}{d t}=-\sum_{\text {jumps }} s f\left(v_{+}, v_{-}\right)-\left.q(U)\right|_{x_{1}} ^{x_{2}}
$$


where $\left.q(U)\right|_{x_{1}} ^{x_{2}}$ is the entropy flux and $f$ is defined by

$$
f\left(v_{+}, v_{-}\right)=\int_{v_{-}}^{v_{+}} \sigma(v) d v-\frac{1}{2}\left\{\sigma\left(v_{+}\right)+\sigma\left(v_{-}\right)\right\}\left(v_{+}-v_{-}\right)
$$

which is called the driving traction in [1], [2]; the thermodynamic account of $f$ will be given in Appendix A. Naturally, we expect that the local entropy (1.9) is decreasing in time, which is equivalent to

$$
s f\left(v_{+}, v_{-}\right) \geq 0
$$

at every discontinuity. At a shock wave, the Lax entropy condition implies the above inequality. At a phase boundary, we adopt Abeyaratne-Knowles' kinetic condition, i.e., there exists a nondecreasing function $\Phi(f)$ satisfying $\Phi(0)=0$ such that the speed of the discontinuity is function of the driving traction:

$$
s=\Phi(f) .
$$

This condition obviously implies (1.11) at the phase boundary. They also assumed that no new phase occurs from any point in the interior of the $\alpha$ or $\beta$-phase, which is called the nucleation condition.

In this paper, we say that a solution is admissible if it satisfies both the kinetic condition and the nucleation condition. Our aim is to study the existence and large time stability of an admissible global in time solution involving a single propagating phase boundary that is obtained by an initial perturbation of the Maxwell states. We shall show that this perturbation problem is stable if we assume that $\Phi$ is a $C^{2}$-function and

$$
\Phi^{\prime}(0)>0
$$

In the following argument, we shall always assume that $\Phi$ satisfies the above condition (1.13) which is not repeatedly stated.

The Riemann problem for (1.1) has been extensively studied in James [15], Hattori [13], Abeyaratne and Knowles [2], LeFloch [18], Corli [6], and Corli and Tougeron [7]. The following theorem is the basis of the present study.

THEOREM 1.1. If $\left|U_{L}-U_{m}\right|,\left|U_{R}-U_{m}^{*}\right|$ are sufficiently small, then there exists a unique admissible solution which consists of 4 constant regions connected by rarefaction waves, shock waves and a phase boundary. Moreover, these constant states are differentiable with respect to the initial data $U_{L}, U_{R}$.

Although, this theorem is contained in the main results of [6] and [7], in order to show the significance of the kinetic condition, we shall give its proof in the next section.

These solutions are used to construct approximate solutions with general initial data (1.4). We obtain global solutions using wave-front tracking method (Bressan [4], Chern [5], Risebro [21]).

THEOREM 1.2. Suppose that the initial perturbation is sufficiently small in total variation, i.e., the quantity:

$$
T . V .\left.\left(U_{L}(x)-U_{m}\right)\right|_{x<0}+T . V .\left.\left(U_{R}(x)-U_{m}^{*}\right)\right|_{x>0}
$$

is sufficiently small. Then there exists a weak global solution with a single phase boundary which is Lipschitz continuous curve in $(x, t)$-space. 
For the single phase boundary arising the tri-linear material ([2]), LeFloch [18] has already established the above existence theorem using the Glimm-Lax theory [12]. Since our system is genuinely nonlinear in hyperbolic regions, cancellation and confluence of waves occur ([12], [19], [20]). In order to show the large-time stability, we have to prove the interaction estimates expressing these phenomena. Hattori [14], also employing the Glimm method, studied the similar problem whose approximate solutions are constructed so as to satisfy the entropy rate admissibility condition of Dafermos [9] and showed that these approximate solutions converge to a global solution. Our scheme is briefly accounted in section 3 and the interaction estimates needed are carried out in sections 4 and 5; these interaction estimates (Lemmas 5.1 and 5.2) are decisive in this paper. Although we have a global in time phase boundary, we do not know if it is admissible in any sense. In [18], LeFloch formulated a weak version of the kinetic condition and showed that the phase boundary obtained satisfies this weak kinetic condition. It is notable that Corli-Tougeron [7] obtained a weak global solutions which are admissible in this weak sense. In section 6 of this paper, also using the Glimm-Lax theory, we study the regularity of the solution at the phase boundary and show that the kinetic condition (1.12) holds almost everywhere: our phase boundary is admissible in the following sense.

THEOREM 1.3. The phase boundary $x=\chi(t)$ is subsonic, and $U(x, t) \in \Omega_{\alpha}$ for $x<\chi(t)$ and $U(x, t) \in \Omega_{\beta}$ for $x>\chi(t)$. The limit:

$$
\lim _{\delta \rightarrow \pm 0} U(\chi(t)+\delta, t)=U_{ \pm}(t)
$$

exists except for countable $t$ at the phase boundary. Moreover, the Rankine-Hugoniot conditions (1.6) and the kinetic condition

$$
\dot{\chi}(t)=\Phi\left(f\left(v_{+}(t), v_{-}(t)\right)\right.
$$

holds at these points.

Finally, we can show that the single phase boundary is asymptotically stable, following the argument in Liu [20] and Asakura [3].

THEOREM 1.4. Suppose that the initial data satisfy

$$
U(x, 0)= \begin{cases}U_{m} & \text { for } x<-M \\ U_{m}^{*} & \text { for } x>M \quad(M>0)\end{cases}
$$

and the total variation of the initial perturbation is sufficiently small. Then the speed and the states both side of the phase boundary approach those of the Maxwell phase boundary at the rate $t^{-3 / 2}$ and the total variation of the solution outside of the phase boundary approaches zero at the rate $t^{-1 / 2}$.

2. The Riemann problem. The Riemann problem is the Cauchy problem with the discontinuous initial data:

$$
(v(x, 0), u(x, 0))= \begin{cases}\left(v_{L}, u_{L}\right) & \text { for } x<0 \\ \left(v_{R}, u_{R}\right) & \text { for } x>0\end{cases}
$$

$\left(v_{L}, u_{L}, v_{R}, u_{R}\right.$ : constants). The system of conservation laws is invariant under the scale transformation

$$
(x, t) \rightarrow(\alpha x, \alpha t), \quad \alpha>0
$$


that is: if $U(x, t)$ is a solution of the conservation laws $(1.10)$, then $V(x, t)=U(\alpha x, \alpha t)$ is also a solution. A solution invariant under the scale transformation

$$
U(\alpha x, \alpha t)=U(x, t)
$$

is called a self-similar solution, which defines the asymptotic behavior of the weak solution (Liu [19]). Such solutions must satisfy the initial condition of the form (2.1). Hence the Riemann problem is important in our study.

For $\left(v_{0}, u_{0}\right) \in \Omega_{\alpha} \cup \Omega_{\beta}$, the integral curve of the $j$-characteristic field $(j=1,2)$ passing through $\left(v_{0}, u_{0}\right)$ is expressed as

$$
\begin{cases}u-u_{0}=\int_{v_{0}}^{v} \sqrt{\sigma^{\prime}(w)} d w, & 1-\text { field } \\ u-u_{0}=-\int_{v_{0}}^{v} \sqrt{\sigma^{\prime}(w)} d w, & 2-\text { field }\end{cases}
$$

These curves define rarefaction waves. Here, for simplicity, we only treat the case: $\sigma^{\prime \prime}(v)<0$ in $\Omega_{\alpha}$ and $\sigma^{\prime \prime}(v)>0$ in $\Omega_{\beta} ;$ in this case $\left(v_{0}, u_{0}\right)$ and $(v, u)$ are connected by

$$
\begin{array}{lll}
1 \text { - rarefaction wave: } & v>v_{0} \text { in } \Omega_{\alpha}, \quad v<v_{0} \text { in } \Omega_{\beta}, \\
2 \text {-rarefaction wave: } & v<v_{0} \text { in } \Omega_{\alpha}, \quad v>v_{0} \text { in } \Omega_{\beta} .
\end{array}
$$

Similarly, the Hugoniot loci are

$$
\begin{cases}u-u_{0}=\sqrt{\frac{\sigma(v)-\sigma\left(v_{0}\right)}{v-v_{0}}}\left(v-v_{0}\right), & 1-\text { field } \\ u-u_{0}=-\sqrt{\frac{\sigma(v)-\sigma\left(v_{0}\right)}{v-v_{0}}}\left(v-v_{0}\right), & 2-\text { field }\end{cases}
$$

and $\left(v_{0}, u_{0}\right)$ and $(v, u)$ are connected by

$$
\begin{array}{lll}
1 \text { - shock wave: } & v<v_{0} \text { in } \Omega_{\alpha}, \quad v>v_{0} \text { in } \Omega_{\beta}, \\
2 \text { - shock wave: } & v>v_{0} \text { in } \Omega_{\alpha}, \quad v<v_{0} \text { in } \Omega_{\beta} .
\end{array}
$$

First suppose that $U_{L}=\left(v_{L}, u_{L}\right)$ and $U_{R}=\left(v_{R}, u_{R}\right)$ are contained in the same phase. The Lax existence theorem ([16]) says that

THEOREM 2.1. If $U_{L}, U_{R}$ belong to a certain domain $\Omega$ in either phase and $\left|U_{L}-U_{R}\right|$ is sufficiently small, then there exists a self-similar solution which consists of 3 constant regions $U_{L}, U_{M}, U_{R}$ connected by rarefaction waves and shock waves. Moreover, as long as the intermediate constant state $U_{M}$ is restricted to $\Omega$, the solution of this form is unique and $U_{M}$ is differentiable with respect to $U_{L}, U_{R}$.

Next suppose that $U_{L}=\left(v_{L}, u_{L}\right)$ and $U_{R}=\left(v_{R}, u_{R}\right)$ are contained in small neighborhoods of the Maxwell states $U_{m}=\left(v_{m}, u_{m}\right)$ and $U_{m}^{*}=\left(v_{m}, u_{m}^{*}\right)$, respectively.

Proposition 2.1. If $\left|U_{m}-U_{L}\right|$ and $\left|U_{m}^{*}-U_{R}\right|$ are sufficiently small, then there exists a self-similar solution which consists of 4 constant regions $U_{L}, U_{-} \in \Omega_{\alpha}$ and $U_{+}, U_{R} \in \Omega_{\beta}$ where $U_{L}, U_{-}$are connected by a 1-rarefaction wave or shock wave in $\Omega_{\alpha}, U_{-}, U_{+}$by a single phase boundary and $U_{+}, U_{R}$ by a 2-rarefaction wave or 
shock wave in $\Omega_{\beta}$. However the solution of this form is not unique and these solutions constitute a one parameter family.

Proof. First, we note that the gradient of the 1-wave curve through $U_{L}$ at this point is $\sqrt{\sigma^{\prime}\left(v_{L}\right)}>0$. Let $U_{-}$be a state on the 1 -wave curve issuing from $U_{L}$ (the combination of the integral curve and the Hugoniot locus through $U_{L}$ ). We find a unique state $U_{-}^{*}=\left(v_{-}^{*}, u_{-}^{*}\right)$ such that

$$
\sigma\left(v_{-}\right)=\sigma\left(v_{-}^{*}\right), \quad u_{-}=u_{-}^{*} .
$$

Let $U_{+}=\left(v_{+}, u_{+}\right)$be a state in $\Omega_{\beta}$ which is connected to $U_{-}$by a phase boundary. Since the propagation speed $s$ of the phase boundary is expressed as

$$
s^{2}=\frac{\sigma\left(v_{+}\right)-\sigma\left(v_{-}\right)}{v_{+}-v_{-}} \geq 0,
$$

$v_{+}$must satisfy

$$
v_{+} \geq v_{-}^{*}
$$

It follows from (2.3) that

$$
\begin{aligned}
\sigma\left(v_{+}\right)-\sigma\left(v_{-}^{*}\right) & =\sigma\left(v_{+}\right)-\sigma\left(v_{-}\right) \\
& =s^{2}\left(v_{-}^{*}-v_{-}\right)+s^{2}\left(v_{+}-v_{-}^{*}\right) .
\end{aligned}
$$

Hence

$$
v_{+}-v_{-}^{*}=\frac{v_{-}^{*}-v_{-}}{\sigma^{\prime}\left(v_{-}^{*}\right)} s^{2}+O\left(s^{4}\right) .
$$

In the same way, we find by the Rankine-Hugoniot condition (1.6) that

$$
\begin{aligned}
u_{+}-u_{-}^{*} & =-s\left(v_{-}^{*}-v_{-}\right)-s\left(v_{+}-v_{-}^{*}\right) \\
& =-s\left(v_{-}^{*}-v_{-}\right)+O\left(s^{3}\right) .
\end{aligned}
$$

Thus, regarding $s$ as a parameter, we can see that $U_{+}$constitutes a regular curve through $U_{-}^{*}$ at which the tangent is perpendicular to the segment connecting $U_{-}$and $U_{-}^{*}$. Since the gradient of 2 -wave curve through $U_{+}$at this point is $-\sqrt{\sigma^{\prime}\left(v_{+}\right)}<0$; hence transversal to the curve defined by (2.5) and (2.6). Then we find that, for any $U_{-}$close to $U_{m}$, there exist a unique $U_{+}$such that $U_{+}$and $U_{R}$ are connected by a 2 -wave. Above proof also shows that we have 1-dimensional freedom for choosing $U_{-}$. ए

Now we consider the kinetic condition

$$
s=\Phi\left(f\left(v_{+}, v_{-}\right)\right)
$$

where we assume that

$$
\Phi(0)=0, \quad \Phi^{\prime}(0)>0 .
$$

At $v_{-}=v_{m}, v_{+}=v_{m}^{*}$, both (2.3) and (2.7) hold with $s=0$. We shall show that these conditions determine $v_{+}$as a function of $v_{-}$. Differentiating (2.3) and (2.7), we have

$$
d s=\frac{1}{2}\left(v_{+}-v_{-}\right) \Phi^{\prime}(f)\left\{\left(s^{2}-\sigma^{\prime}\left(v_{-}\right)\right) d v_{-}+\left(s^{2}-\sigma^{\prime}\left(v_{+}\right)\right) d v_{+}\right\}
$$




$$
2 s d s=\frac{1}{v_{+}-v_{-}}\left\{\left(s^{2}-\sigma^{\prime}\left(v_{-}\right)\right) d v_{-}-\left(s^{2}-\sigma^{\prime}\left(v_{+}\right)\right) d v_{+}\right\}
$$

Since $\left(s^{2}-\sigma^{\prime}\left(v_{+}\right)\right)\left(s^{2}-\sigma^{\prime}\left(v_{-}\right)\right) \Phi^{\prime}(f) \neq 0$ at $s=0, v_{+}$and $v_{-}$are functions of $s$ in a neighborhood of $s=0$ and

$$
\begin{aligned}
\frac{d v_{-}}{d s} & =\frac{1+s\left(v_{+}-v_{-}\right)^{2} \Phi^{\prime}(f)}{\left(v_{+}-v_{-}\right)\left(s^{2}-\sigma^{\prime}\left(v_{-}\right)\right) \Phi^{\prime}(f)} \\
\frac{d v_{+}}{d s} & =\frac{1-s\left(v_{+}-v_{-}\right)^{2} \Phi^{\prime}(f)}{\left(v_{+}-v_{-}\right)\left(s^{2}-\sigma^{\prime}\left(v_{+}\right)\right) \Phi^{\prime}(f)} .
\end{aligned}
$$

Moreover, $d v_{-} / d s \neq 0$ at $s=0$ says that $v_{+}$and $s$ are functions of $v_{-}$. Thus we have proved

LEMMA 2.1. If $\Phi(0)=0$ and $\Phi^{\prime}(0)>0$, then $v_{+}$and $s$ are functions of $v_{-}$in a neighborhood of $v_{m}$ and

$$
\begin{aligned}
& \left.\frac{d v_{+}}{d v_{-}}\right|_{v_{-}=v_{m}}=\frac{\sigma^{\prime}\left(v_{m}\right)}{\sigma^{\prime}\left(v_{m}^{*}\right)}, \\
& \left.\frac{d s}{d v_{-}}\right|_{v_{-}=v_{m}}=-\Phi^{\prime}(0) \sigma^{\prime}\left(v_{m}\right)\left(v_{m}^{*}-v_{m}\right) .
\end{aligned}
$$

We note that the derivative of $v_{+}$does not depend on the particular value of $\Phi^{\prime}(0)$ and coincides with the expression coming from the entropy rate admissibility condition in Hattori [13].

Proof. [Proof of Theorem 1.1] We normalize the characteristic fields $R_{1}(U), R_{2}(U)$ for $\lambda_{1}(U)=-\sqrt{\sigma^{\prime}(v)}, \lambda_{2}(U)=\sqrt{\sigma^{\prime}(v)}$, respectively, so that

$$
R_{1}(U) \cdot \nabla \lambda_{1}=R_{2}(U) \cdot \nabla \lambda_{2}=1 .
$$

For any state $U_{0}$ close to $U_{m}$ or $U_{m}^{*}$, we can define 1 and 2-wave curve through $U_{0}$ parameterized $\epsilon_{1}$ and $\epsilon_{2}$, respectively

$$
U=U_{1}\left(\epsilon_{1} ; U_{0}\right), \quad U=U_{2}\left(\epsilon_{2} ; U_{0}\right) .
$$

By virtue of (2.13), these parameters are expressed as

$$
\epsilon_{j}=\lambda_{j}\left(U_{j}\left(\epsilon_{j} ; U_{0}\right)\right)-\lambda_{j}\left(U_{0}\right), \quad j=1,2 .
$$

Suppose that the state $U_{L}$ is close to $U_{m}$. We set $U_{-}\left(\epsilon_{1} ; U_{L}\right)=U_{1}\left(\epsilon_{1} ; U_{L}\right)$. Since, by Lemma 2.1, admissible $v_{+}$and $s$ are functions of $v_{-}$, we can define a mapping $U_{-}=\left(v_{-}, u_{-}\right) \rightarrow U_{+}\left(U_{-}\right)=\left(v_{+}, u_{+}\right)$to be

$$
\left\{\begin{array}{l}
v_{+}=v_{+}\left(v_{-}\right), \\
u_{+}=u_{-}-s\left(v_{-}\right)\left\{v_{+}\left(v_{-}\right)-v_{-}\right\}
\end{array}\right.
$$

whose Jacobian matrix at $U_{-}=U_{m}$ is

$$
U_{+}^{\prime}\left(U_{m}\right)=\left(\begin{array}{cc}
\sigma^{\prime}\left(v_{m}\right) \sigma^{\prime}\left(v_{m}^{*}\right)^{-1} & 0 \\
\Phi(0) \sigma^{\prime}\left(v_{m}\right)\left(v_{m}^{*}-v_{m}\right)^{2} & 1
\end{array}\right) .
$$


Clearly, this shows that $U_{+}\left(U_{-}\right)$is a diffeomorphism in a neighborhood of $U_{L}$ close to $U_{m}$. Using this mapping, we set

$$
U\left(\epsilon_{1}, \epsilon_{2} ; U_{L}\right)=U_{2}\left(\epsilon_{2} ; U_{+}\left(U_{-}\left(\epsilon_{1} ; U_{L}\right)\right)\right)
$$

which has an expansion

$$
\begin{aligned}
U\left(\epsilon_{1}, \epsilon_{2} ; U_{L}\right) & =U_{+}\left(U_{-}\left(\epsilon_{1} ; U_{L}\right)\right)+\epsilon_{2} R_{2}\left(U_{+}\left(U_{-}\left(\epsilon_{1} ; U_{L}\right)\right)\right)+O\left(\epsilon_{2}^{2}\right) \\
& =U_{+}\left(U_{L}\right)+\epsilon_{1} U_{+}^{\prime}\left(U_{L}\right) R_{1}\left(U_{L}\right)+\epsilon_{2} R_{2}\left(U_{+}\left(U_{L}\right)\right)+O\left(|\epsilon|^{2}\right) .
\end{aligned}
$$

By direct calculations, we have

$$
\begin{aligned}
U_{+}^{\prime}\left(U_{m}\right) R_{1}\left(U_{m}\right) & \propto\left(\begin{array}{c}
\sigma^{\prime}\left(v_{m}^{*}\right)^{-1} \\
\Phi(0) \sqrt{\sigma^{\prime}\left(v_{m}\right)}\left(v_{m}^{*}-v_{m}\right)^{2}
\end{array}\right), \\
R_{2}\left(U_{m}^{*}\right) & \propto\left(\begin{array}{c}
-1 \\
\sqrt{\sigma^{\prime}\left(v_{m}\right)}
\end{array}\right),
\end{aligned}
$$

which shows that these vectors are linearly independent; hence the mapping $\left(\epsilon_{1}, \epsilon_{2}\right) \rightarrow$ $U\left(\epsilon_{1}, \epsilon_{2} ; U_{L}\right)$ is a diffeomorphism for small $\epsilon$ at $U_{L}$ close to $U_{m}$ and its image contains $U_{m}^{*}$. The theorem follows directly from these observations.

3. Wave-front tracking. We carry out the proof Theorem 1.2. First, we construct an approximation of the solution to the Riemann problem. Let $U_{j}(0 \leq j \leq 3)$ be constant states constituting the solution. If two adjoining states $U_{j-1}$ and $U_{j}$ are connected by a shock wave or a phase boundary, we leave them as they are. Suppose that these constant state are connected by a rarefaction wave and denote by $U_{j}\left(\epsilon ; U_{0}\right)$ the $j$-rarefaction curve issuing from $U_{0}$. For any positive $h$, we choose an integer $k$ such that $k h \leq \epsilon_{j}<(k+1) h$ and define constant states $U_{j}^{(l)}(0 \leq l \leq k)$ by the partition

$$
U_{j}^{(0)}=U_{j-1}, \quad U_{j}^{(l)}=U_{j}\left(l h ; U_{j-1}\right)=U_{j}\left(h ; U_{j}^{(l-1)}\right) .
$$

The approximation $U^{h}(x, t)$ is defined by

$$
U^{h}(x, t)=U_{j}^{(l)}, \quad \lambda_{j}\left(U_{j}^{(l)}\right) t<x<\lambda_{j}\left(U_{j}^{(l+1)}\right) t, \quad l=0,1, \ldots, k
$$

$\left(U_{j}^{(k+1)}=U_{j}\right)$. This approximation consists of constant states separated by a phase boundary and discontinuities propagating in the $j$-th characteristic direction; these discontinuities are simply called $j$-waves and the approximation $U^{h}$ is called the $h$ approximation. Obviously, $U^{h}$ converges to $U$ in uniform convergence. By assuming the support of $\phi$ is contained in $[0, T]$, the truncation error as a weak solution is expressed as

$$
\iint_{R \times R_{+}}\left\{U^{h} \phi_{t}+F\left(U^{h}\right) \phi_{x}\right\} d x d t+\int_{R} U_{0}(x) \phi(x, 0) d x=O(h T) \sum_{\epsilon_{j}>0} \epsilon_{j} .
$$

Now we construct an approximate solution for general initial data following Risebro [21]. For any positive $h$, we choose a sequence $\left\{x_{j}\right\}_{j=1}^{N_{0}}$ in $R$ such that

$$
\begin{aligned}
x_{1} & =\inf \left\{x ;\left|U_{0}(x)-U_{m}\right|>h\right\}, \\
x_{n+1} & =\inf \left\{x ; x>x_{n} \text { and }\left|U_{0}(x)-\lim _{x \rightarrow x_{n}+0} U(x)\right|>h\right\} .
\end{aligned}
$$


We define the approximation of the initial data by step functions:

$$
U_{0}^{h}(x)= \begin{cases}U_{m}, & x<x_{1}, \\ U_{0}\left(x_{n}\right), & x_{n} \leq x<x_{n+1}, \\ U_{m}^{*}, & x \geq x_{N_{0}}\end{cases}
$$

so that

$$
\sup _{x \neq x_{n}}\left|U_{0}^{h}(x)-U_{0}(x)\right| \leq h .
$$

At each point of discontinuity $x=x_{n}$, we solve the Riemann problem with the initial data $U_{L}=U_{0}^{h}\left(x_{n}-0\right), U_{R}=U_{0}^{h}\left(x_{n}+0\right)$. If both $U_{L}$ and $U_{R}$ belong to the same phase, we adopt the Lax solution, because we assume the nucleation condition; if $U_{L} \in \Omega_{\alpha}$ and $U_{R} \in \Omega_{\beta}$, we construct the admissible solution following the way in section 2 . We define the approximate solution by combining the $h$-approximations $(3.2$ jof these solutions. This approximate solution, denoted by $U^{h}(x, t)$ and called also the $h$ approximation, is defined as long as the neighboring waves of the $h$-approximations collide at $t_{1}>0$. At $t=t_{1}$, since the approximation $U^{h}\left(x, t_{1}-0\right)$ is also a step function, we can construct a $h$-approximation by solving the Riemann problem. Then we can extend the approximation to the next collision time $t=t_{2}>t_{1}$. Repeating this construction, we obtain the approximate solution. Let $t_{m}$ denote the $m$-th collision time and

$$
T=\lim _{m \rightarrow \infty} t_{m}
$$

If $T=\infty$, the approximate solution is global in time; however, this is not always the case. If $T<\infty$, we slightly change the definition of the approximation by neglecting small waves which are produced by the repeated collision. In order to carry out this process, we have to show interaction estimates.

4. Local interaction of waves. Let $x=x_{l}$ be the point of collision at $t=t_{m}$ in the hyperbolic region $\Omega_{\alpha} \cup \Omega_{\beta}$ where $p$ waves collide; we set $\mathrm{P}_{l, m}=\left(x_{l}, t_{m}\right)$ and sometimes denote by $\mathrm{P}$ for simplicity. We call these waves the incoming waves which are denoted by $\alpha=\left(\alpha_{2}^{(1)}, \ldots, \alpha_{2}^{\left(p_{2}\right)}, \alpha_{1}^{(1)} \ldots, \alpha_{1}^{\left(p_{1}\right)}\right)$ from left to right $\left(p=p_{1}+p_{2}\right)$. Here the index $i=1,2$ says that $\alpha_{i}^{(k)}$ is an $i$-wave. The elementary waves issuing from $\mathrm{P}$, denoted by $\epsilon=\left(\epsilon_{1}, \epsilon_{2}\right)$, are called the outgoing waves. We say in short that $\alpha$ interact at $\mathrm{P}$ and produce $\epsilon$. The amplitude of outgoing waves $\epsilon$ are $C^{2}$-functions of $\alpha$ with Lipschitz continuous third derivatives. We define the amount of interaction at the point of collision $\mathrm{P}$ as the following:

$$
Q(\mathrm{P})=Q_{d}(\mathrm{P})+Q_{s}(\mathrm{P})
$$

where

$$
\begin{aligned}
Q_{d}(\mathrm{P}) & =\sum_{\substack{1 \leq k \leq p_{1} \\
1 \leq l \leq p_{2}}}\left|\alpha_{1}^{(k)} \alpha_{2}^{(l)}\right|, \\
Q_{s}(\mathrm{P}) & =\sum_{\substack{i=1,2 \\
k \neq l}} Q_{s}\left(\alpha_{i}^{(k)} \alpha_{i}^{(l)}\right),
\end{aligned}
$$




$$
Q_{s}(\alpha, \beta)= \begin{cases}0 & \alpha \geq 0, \beta \geq 0 \\ |\alpha|^{3} & |\alpha| \leq|\beta|, \beta \geq 0, \alpha<0 \\ |\alpha||\beta|^{2} & |\alpha| \leq|\beta|, \beta<0 \\ |\beta||\alpha|^{2} & |\beta| \leq|\alpha|, \alpha<0 \\ |\beta|^{3} & |\beta| \leq|\alpha|, \alpha \geq 0, \beta<0\end{cases}
$$

or

$$
= \begin{cases}0 & \alpha \geq 0, \beta \geq 0 \\ |\alpha \beta|(|\alpha|+|\beta|) & \text { otherwise }\end{cases}
$$

(cf. Liu [20] Theorem 2.2). The following estimates shows that the interaction among small waves of different characteristic family produces second order small waves and waves of the same family the third order small waves; proof is carried out in the same manner as Glimm [11] and Liu [20].

LEMMA 4.1 (Local interaction estimate). If the amplitude of incoming waves is sufficiently small, then it follows that

$$
\epsilon_{i}=\sum_{k} \epsilon_{i}^{(k)}=\sum_{k=1}^{p_{i}} \alpha_{i}^{(k)}+O(1) Q(\mathrm{P}), \quad i=1,2 .
$$

Here the first sum denotes the partition of the outgoing $i$-wave; $O(1)$ depends only on the system and the two constant states connected by the incoming waves.

In [5], Chern studied the wave interaction problem where a single relatively strong shock wave is involved. In his case the above interaction term is no more quadratic. He singled out the scattered wave, which is of order 1, and showed that pure interaction term is quadratic. We use his idea to study the wave interaction at the phase boundary. Let $\mathrm{P}$ be a point of collision involving a phase boundary. Suppose that a phase boundary $\pi$ and $\beta=\left(\beta_{2}^{(1)}, \ldots, \beta_{2}^{\left(p_{2}\right)}, \beta_{1}^{(1)} \ldots, \beta_{1}^{\left(p_{1}\right)}\right)$ enter $\mathrm{P}$, and generate $\pi^{\prime}$ and $\beta^{\prime}=\left(\beta_{1}^{\prime}, \beta_{2}^{\prime}\right)$. Here we set $Q(\mathrm{P})=0$. Instead, we denote by $A(\mathrm{P})$ the total amount of incoming waves:

$$
A(\mathrm{P})=\sum_{\substack{i=1,2 \\ 1 \leq k_{i} \leq p_{i}}}\left|\beta_{i}^{\left(k_{i}\right)}\right| .
$$

Since the amplitude of outgoing waves $\beta^{\prime}$ is $C^{2}$-functions of $\beta$, obviously we have (see [5] Lemma 3.3).

LEMMA 4.2 (Local interaction estimate). If the amplitude of incoming waves is sufficiently small, then it follows that

$$
\pi^{\prime}=\pi+O(1) A(\mathrm{P}), \quad \beta_{i}^{\prime}=O(1) A(\mathrm{P}), \quad i=1,2
$$

Here $O(1)$ depends only the two constant states connected by the incoming waves and the system.

5. Global interaction of waves. In this section we shall show the precise estimates for the amount of interaction involving rarefaction waves, shock waves and a single phase boundary. We say that a continuous curve $J$ is an approximate spacelike curve, if $J$ is composed of space-like segments. These curves are partially ordered 
by denoting $J_{2}>J_{1}$ if every point of $J_{1}$ lies on $J_{2}$ or between $J_{2}$ and $t=0$. Let $J$ be an approximate space-like curve and $W(J)$ the collection of (the approximation of) rarefaction waves and shock waves crossing $J$. We denote by $W^{+}(J)$ and $W^{-}(J)$ the set of (the approximation of) rarefaction waves and shock waves, respectively, crossing $J$. Also $W_{A}(J)$ is the set of these waves crossing $J$ and approaching the phase boundary. The single phase boundary crossing $J$ is denoted by $\pi(J)$. We set

$$
\begin{aligned}
L(J) & =L^{+}(J)+L^{-}(J), \\
L^{ \pm}(J) & =\sum_{\alpha \in W^{ \pm}(J)}|\alpha|, \\
L_{A}(J) & =\sum_{\alpha \in W_{A}(J)}|\alpha| .
\end{aligned}
$$

We define the interaction potential as the following.

$$
\widehat{Q}_{s}(J)=Q_{s}(J)+K Q_{a}(J)+K^{2} Q_{a a}(J)
$$

where

$$
\begin{aligned}
Q_{s}(J)=\sum & \left\{|\alpha|^{3} ; \alpha \in W^{-}(J)\right\} \\
& +4 \sum\left\{|\beta \gamma||\beta+\gamma| ; \beta, \gamma \in W^{-}(J), \beta \neq \gamma \text { and of the same family }\right\} \\
& +8 \sum\left\{|\epsilon \eta \zeta| ; \epsilon, \eta, \zeta \in W^{-}(J), \text { distinct and of the same family }\right\}
\end{aligned}
$$

or

$$
\begin{aligned}
& \qquad\{|\beta \gamma|(|\beta|+|\gamma|) ; \beta, \gamma \text { are any } j \text {-waves in } W(J) ; \text { not both of } \\
& \beta, \gamma \text { are } j \text {-rarefaction waves }\} \\
& Q_{a}(J)=\sum\left\{|\alpha \beta| ; \alpha \in W_{A}(J), \beta \in W(J) \text { and } \alpha \neq \beta\right\} \\
& Q_{a a}(J)=\sum\left\{|\alpha \beta| ; \alpha, \beta \in W_{A}(J)\right\}
\end{aligned}
$$

We adapt $\widetilde{Q}(J)_{o o}$ in [5] according with Liu's observation that the interaction of the waves of the same family produces the waves of order 3 .

Now we estimate the total amount of interaction produced by the waves of the same family with the aid of the following lemma.

Lemma 5.1. Let $J_{1}, J_{2}$ be two approximate space-like curves such that $J_{2}>J_{1}$ and $\mathrm{P}$ a single point of interaction between them. If $K>2 M$ and $L\left(J_{1}\right) \leq 1$, then it follows that

$$
Q_{s}(\mathrm{P}) \leq \widehat{Q}_{s}\left(J_{1}\right)-\widehat{Q}_{s}\left(J_{2}\right)+M\left(1+K+K^{2}\right) L\left(J_{1}\right) Q(\mathrm{P}) .
$$

Here $M$ stands for $O(1)$ in Proposition 4.1.

Proof. We discuss only $Q_{s}$ defined by (4.3); in the case (4.4), estimates are carried out in the same way. Suppose that $\alpha$ enter and $\alpha^{\prime}$ leaves. 
Case 1. The phase boundary does not enter P. Estimates of $Q_{s}(J)$ : we may assume that each $\alpha^{\prime}, \alpha$ consists only of $j$-wave. If $\alpha^{\prime} \geq 0$, obviously we have

$$
Q_{s}\left(J_{2}\right) \leq Q_{s}\left(J_{1}\right)-Q_{s}(\mathrm{P})
$$

Assume that $\alpha^{\prime}<0$ and let $\epsilon, \zeta \in W^{-}\left(J_{2}\right)$ denote $j$-waves not entering P. If all incoming waves are shock waves, we have

$$
\left|\alpha^{\prime}\right|=\sum_{1 \leq k \leq p}\left|\alpha^{(k)}\right|+O(1) Q(\mathrm{P})
$$

Hence

$$
\begin{aligned}
\left|\alpha^{\prime}\right|^{3}+4 \sum_{\epsilon \neq \alpha^{\prime}}\left|\epsilon \alpha^{\prime}\right|\left|\epsilon+\alpha^{\prime}\right|+8 \sum_{\epsilon, \eta \neq \alpha^{\prime}}\left|\epsilon \eta \alpha^{\prime}\right|=\sum_{k}\left|\alpha^{(k)}\right|^{3}+4 \sum_{k, \epsilon \neq \alpha}\left|\epsilon \alpha^{(k)}\right|\left|\epsilon+\alpha^{(k)}\right| \\
+8 \sum_{k, \epsilon, \eta \neq \alpha}\left|\epsilon \eta \alpha^{(k)}\right|+3 \sum_{k<l}\left|\alpha^{(k)} \alpha^{(l)}\right|\left|\alpha^{(k)}+\alpha^{(l)}\right| \\
+6 \sum_{k<l<m}\left|\alpha^{(k)} \alpha^{(l)} \alpha^{(m)}\right|+8 \sum_{\substack{k<l \\
\epsilon \neq \alpha}}\left|\epsilon \alpha^{(k)} \alpha^{(l)}\right|+O(1) L^{-}\left(J_{1}\right) Q(\mathrm{P})
\end{aligned}
$$

Thus, we obtain

$$
Q_{s}\left(J_{2}\right) \leq Q_{s}\left(J_{1}\right)-Q_{s}(\mathrm{P})+M L^{-}\left(J_{1}\right) Q(\mathrm{P})
$$

If a certain $\alpha^{\left(k_{0}\right)}$ is a rarefaction, we find that

$$
\left|\alpha^{\prime}\right| \leq \sum_{\substack{1 \leq k \leq p \\ k \neq k_{0}}}\left|\alpha^{(k)}\right|+M Q(\mathrm{P})
$$

Thus in any case, we obtain (5.3).

Estimates of $Q_{a}(J)$ :

$$
\begin{aligned}
Q_{a}\left(J_{2}\right) & =\sum_{\substack{\epsilon \neq \alpha^{\prime} \\
\epsilon \in W_{A}\left(J_{1}\right)}}\left|\epsilon \alpha^{\prime}\right|+\left(\text { terms not involving } \alpha^{\prime}\right) \\
& \leq \sum_{\substack{k, \epsilon \neq \alpha^{(k)} \\
\epsilon \in W_{A}\left(J_{1}\right)}}\left|\epsilon \alpha^{(k)}\right|+M L_{A}\left(J_{1}\right) Q(\mathrm{P})+\left(\text { terms not involving } \alpha^{(k)}\right) \\
& \leq Q_{a}\left(J_{1}\right)+M L_{A}\left(J_{1}\right) Q(\mathrm{P}) .
\end{aligned}
$$

Estimates of $Q_{a a}(J)$ : as above we have

$$
Q_{a a}\left(J_{2}\right) \leq Q_{a a}\left(J_{1}\right)+M L_{A}\left(J_{1}\right) Q(\mathrm{P}) .
$$

Hence we obtain

$$
\widehat{Q}\left(J_{2}\right) \leq \widehat{Q}\left(J_{1}\right)-Q_{s}(\mathrm{P})+M\left(1+K+K^{2}\right) L\left(J_{1}\right) Q(\mathrm{P}) .
$$


Case 2. The phase boundary enters $\mathbf{P}$. Let the phase boundary $\pi$ and $\beta=$ $\left(\beta_{2}^{(1)}, \ldots, \beta_{2}^{\left(p_{2}\right)}, \beta_{1}^{(1)}, \ldots, \beta_{1}^{\left(p_{1}\right)}\right)$ enter $\mathrm{P}$, and generate $\pi^{\prime}$ and $\beta^{\prime}=\left(\beta_{1}^{\prime}, \beta_{2}^{\prime}\right)$. Omitting the suffix 1, 2 for simplicity, we have by Lemma 4.2

$$
\beta^{\prime}=O(1) A(\mathrm{P}), \quad A(\mathrm{P})=\sum_{k}\left|\beta^{(k)}\right| .
$$

Estimates of $Q_{s}(J)$ : if $\beta^{\prime} \geq 0$, obviously we have

$$
Q_{s}\left(J_{2}\right) \leq Q_{s}\left(J_{1}\right)
$$

Assume that $\beta^{\prime}<0$ and let $\epsilon, \eta \in W^{-}\left(J_{2}\right)$ denote $j$-waves not entering P. Since $L\left(J_{1}\right) \leq 1$, we have

$$
\left|\beta^{\prime}\right|^{3}+4 \sum_{\epsilon \neq \beta^{\prime}}\left|\epsilon \beta^{\prime}\right|\left|\epsilon+\beta^{\prime}\right|+8 \sum_{\epsilon, \eta \neq \beta^{\prime}}\left|\epsilon \eta \beta^{\prime}\right|=O(1) \sum_{\epsilon \neq \beta}|\epsilon| A(\mathrm{P})+O(1) A(\mathrm{P})^{2} .
$$

Thus we obtain

$$
Q_{s}\left(J_{2}\right) \leq Q_{s}\left(J_{1}\right)+M \sum_{\epsilon \neq \beta}|\epsilon| A(\mathrm{P})+M \sum_{\epsilon \in W_{A}\left(J_{1}\right)}|\epsilon| A(\mathrm{P}) .
$$

Estimates of $Q_{a}(J)$ : as above we have

$$
\begin{aligned}
Q_{a}\left(J_{2}\right) & =\sum_{\epsilon \in W_{A}\left(J_{1}\right)}\left|\epsilon \beta^{\prime}\right|+\left(\text { the terms not involving } \beta^{\prime}\right) \\
& \leq M \sum_{\substack{\epsilon \in W_{A}\left(J_{1}\right) \\
\epsilon \neq \beta}}|\epsilon| A(\mathrm{P})+(\text { the terms not involving } \beta) \\
& \leq Q_{a}\left(J_{1}\right)-\sum_{\epsilon \neq \beta}|\epsilon| A(\mathrm{P})+M \sum_{\substack{\epsilon \in W_{A}\left(J_{1}\right) \\
\epsilon \neq \beta}}|\epsilon| A(\mathrm{P}) .
\end{aligned}
$$

Estimates of $Q_{a a}(J)$ : clearly we have

$$
Q_{a a}\left(J_{2}\right) \leq Q_{a a}\left(J_{1}\right)-\sum_{\epsilon \in W_{A}\left(J_{1}\right)}|\epsilon| A(\mathrm{P}) .
$$

Thus combining the above estimates and setting $K>2 M$, we obtain

$$
\begin{aligned}
& \widehat{Q}_{s}\left(J_{2}\right) \leq \widehat{Q}_{s}\left(J_{1}\right)+(M-K) \sum_{\substack{\epsilon \in W\left(J_{1}\right) \\
\epsilon \neq \beta}}|\epsilon| A(\mathrm{P}) \\
&+\left(M+M K-K^{2}\right) \sum_{\epsilon \in W_{A}\left(J_{1}\right)}|\epsilon| A(\mathrm{P}) \leq \widehat{Q}_{s}\left(J_{1}\right),
\end{aligned}
$$

which proves the lemma.

In order to estimate $Q(\mathrm{P})$, we define another interaction potential:

$$
\widehat{Q}_{d}(J)=Q_{d}(J)+K Q_{d a}(J)
$$

where

$$
Q_{d}(J)=\sum\{|\alpha \beta| ; \alpha, \beta \in W(J) \text { and } \alpha \text { is any } i \text {-wave }
$$




$$
\begin{aligned}
& \text { lying to the left of a } j \text {-wave } \beta \text { with } i>j\}, \\
& Q_{d a}(J)=\sum\left\{|\alpha \beta| ; \alpha, \beta \in W_{A}(J) \text { and } \alpha \neq \beta\right\} .
\end{aligned}
$$

The next lemma shows that the amount of interaction produced by the waves of the different family is estimated in the same manner as (5.2). We set

$$
Q(J)=\widehat{Q}_{d}(J)+\widehat{Q}_{s}(J) .
$$

LEMMA 5.2. Let $Q(\mathrm{P})$ be the amount of interaction at $\mathrm{P}$ defined by (4.1) and $A(\mathrm{P})$ the amount of waves incoming to $\mathrm{P}$ defined by (4.6). Then it follows that

$$
\begin{aligned}
& Q(\mathrm{P}) \leq Q\left(J_{1}\right)-Q\left(J_{2}\right)+M\left(1+K+K^{2}\right) L\left(J_{1}\right) Q(\mathrm{P}), \\
& A(\mathrm{P}) \leq L_{A}\left(J_{1}\right)-L_{A}\left(J_{2}\right)+M Q(\mathrm{P}) .
\end{aligned}
$$

Here $M$ stands for $O(1)$ in Proposition 4.1.

Proof. In order to prove (5.9), it suffices to show that

$$
\sum_{k, l}\left|\alpha_{1}^{(k)} \alpha_{2}^{(l)}\right| \leq\left(\widehat{Q}_{d}\left(J_{1}\right)-\widehat{Q}_{d}\left(J_{2}\right)\right)+M L\left(J_{1}\right) Q(\mathrm{P}) .
$$

Case 1. The phase boundary does not enter P. Estimates of $Q_{d}(J)$ :

$$
\begin{aligned}
Q_{d}\left(J_{2}\right) & =\sum_{\epsilon_{1} \in W\left(J_{1}\right)}\left|\epsilon_{1} \alpha_{2}^{\prime}\right|+\sum_{\epsilon_{2} \in W\left(J_{1}\right)}\left|\alpha_{1}^{\prime} \epsilon_{2}\right|+\left(\text { terms not involving } \alpha^{\prime}\right) \\
& \leq \sum_{1 \leq k \leq p_{2}}\left|\epsilon_{1} \alpha_{2}^{(k)}\right|+\sum_{1 \leq k \leq p_{1}}\left|\alpha_{1}^{(k)} \epsilon_{2}\right|+M L\left(J_{1}\right) Q(\mathrm{P})+\left(\text { terms not involving } \alpha^{\prime}\right) \\
& \leq Q_{d}\left(J_{1}\right)-\sum_{k, l}\left|\alpha_{1}^{(k)} \alpha_{2}^{(l)}\right|+M L\left(J_{1}\right) Q(\mathrm{P}) .
\end{aligned}
$$

Estimates of $Q_{d a}(J)$ : similarly we have

$$
Q_{d a}\left(J_{2}\right) \leq Q_{d a}\left(J_{1}\right)+M L\left(J_{1}\right) Q(\mathrm{P}) .
$$

Hence we obtain

$$
\widehat{Q}_{d}\left(J_{2}\right) \leq \widehat{Q}_{d}\left(J_{1}\right)-\sum_{k, l}\left|\alpha_{1}^{(k)} \alpha_{2}^{(l)}\right|+M(1+K) L\left(J_{1}\right) Q(\mathrm{P}) .
$$

Case 2. The phase boundary enters P. Estimates of $Q_{d}(J)$ : as above we have

$$
\begin{aligned}
Q_{d}\left(J_{2}\right) & =\sum_{\epsilon_{1} \in W\left(J_{1}\right)}\left|\epsilon_{1} \beta_{2}^{\prime}\right|+\sum_{\epsilon_{2} \in W\left(J_{1}\right)}\left|\beta_{1}^{\prime} \epsilon_{2}\right|+\left(\text { terms not involving } \alpha^{\prime}\right) \\
& \leq M \sum_{\substack{\epsilon \in W_{A}\left(J_{1}\right) \\
\epsilon \neq \beta}}|\epsilon| A(\mathrm{P})+\left(\text { terms not involving } \alpha^{\prime}\right) \\
& \leq Q_{d}\left(J_{1}\right)+M \sum_{\substack{\epsilon_{1} \in W_{A}\left(J_{1}\right), k \\
\epsilon_{1} \neq \beta_{1}}}\left|\epsilon_{1} \beta_{1}^{(k)}\right|+M \sum_{\substack{\epsilon_{2} \in W_{A}\left(J_{1}\right), k \\
\epsilon_{2} \neq \beta_{2}}}\left|\epsilon_{2} \beta_{2}^{(k)}\right| .
\end{aligned}
$$


Estimates of $Q_{d a}(J)$ : clearly we have

$$
Q_{d a}\left(J_{2}\right) \leq Q_{d a}\left(J_{1}\right)-\sum_{\substack{\epsilon \in W_{A}(J), k \\ \epsilon \neq \beta_{1}}}\left|\epsilon \beta_{1}^{(k)}\right|-\sum_{\substack{\epsilon \in W_{A}(J), k \\ \epsilon \neq \beta_{2}}}\left|\epsilon \beta_{2}^{(k)}\right| .
$$

Thus combining the above estimates and setting $K>M$, we obtain (5.12) and hence (5.11).

Estimate of (5.10) is easy: if $\mathrm{P}$ is off the phase boundary, $A(\mathrm{P})=0$ and

$$
L_{A}\left(J_{2}\right) \leq L_{A}\left(J_{1}\right)+M Q(\mathrm{P})
$$

If $\mathrm{P}$ is on the phase boundary, we have

$$
A(\mathrm{P}) \leq L_{A}\left(J_{1}\right)-L_{A}\left(J_{2}\right)
$$

Thus (5.10) follows.

Above two lemmas are the main contributions of this paper. If $L\left(J_{1}\right) \leq \frac{1}{2}(1+$ $K+K^{2}$ ), then the lemmas imply

$$
Q(\mathrm{P}) \leq 2\left\{Q\left(J_{1}\right)-Q\left(J_{2}\right)\right\} .
$$

Suppose that the initial perturbation is sufficiently small so that

$$
L(O) \leq \frac{1}{2}\left(1+K+K^{2}\right) .
$$

Then it follows that for any $J$

$$
\begin{aligned}
\sum_{P} Q(\mathrm{P}) & \leq 2\{Q(O)-Q(J)\}: \text { sum between } O \text { and } J \\
& \leq 2 Q(O) \leq 2 L(O)^{2}
\end{aligned}
$$

Similarly it follows from (5.10) that

$$
\sum_{P} A(\mathrm{P}) \leq L(O)+2 M L(O)^{2}
$$

Moreover

$$
\begin{aligned}
|\pi(J)-\pi(O)| & \leq \sum_{P} A(\mathrm{P}): \text { sum between } O \text { and } J \\
& \leq L(O)+2 M L(O)^{2} .
\end{aligned}
$$

Using the above estimates, we can show a uniform estimate of $L(J)$ in the following way. Let $J_{1}, J_{2}$ be two approximate space-like curves such that $J_{2}>J_{1}$ and $\mathrm{P}$ a single point of interaction between them. By the local interaction estimates (4.5) and (4.7), we have

$$
L\left(J_{2}\right) \leq L\left(J_{1}\right)+M A(\mathrm{P})+M Q(\mathrm{P})
$$

Hence for any $J$

$$
L(J) \leq L(O)+M \sum_{P} A(\mathrm{P})+M \sum_{P} Q(\mathrm{P})
$$




$$
\leq(1+M) L(O)+2 M(1+M) L(O)^{2} .
$$

In this way, we obtain the following a priori estimates

LEMmA 5.3 (Global interaction estimates). If $L(O) \leq \frac{1}{2}\left(1+K+K^{2}\right)$, then it follows that the total amount of interaction and that of waves approaching to the phase boundary are uniformly bounded.

$$
\begin{gathered}
\sum_{P} Q(\mathrm{P}) \leq 2 L(O)^{2}, \quad \sum_{P} A(\mathrm{P}) \leq L(O)+2 M L(O)^{2}, \\
|\pi(J)-\pi(O)| \leq L(O)+2 M L(O)^{2} .
\end{gathered}
$$

Moreover, $L(J)$ has a uniform estimate

$$
L(J) \leq(1+M)\left\{L(O)+2 M L(O)^{2}\right\} .
$$

Proof. [Proof of Theorem 1.2] We can extend the approximate solution beyond $T=\lim _{m \rightarrow \infty} t_{m}$ in the following way. We recall that $\mathrm{P}_{l, m}$ denotes $\left(x_{l}, t_{m}\right)$ where $x=x_{l}$ is the point of collision at $t=t_{m}$. The estimate (5.14) implies

$$
\sum_{l} Q\left(\mathrm{P}_{l, M_{1}}\right)<h \quad \text { for some } M_{1} \text {. }
$$

At $t=t_{M_{1}}$, the approximation of the Riemann problem is so constructed as to consist only of waves whose directions are characteristic directions of the incoming waves; other waves are removed and the constant state at either side of the wave is extended (see Risebro [21] for the details). Repeating this argument, we obtain a sequence of collision times

$$
t_{1}^{(1)}, \ldots, t_{M_{1}}^{(1)}, t_{1}^{(2)}, \ldots, t_{M_{2}}^{(2)}, \ldots, t_{1}^{(k)}, \ldots, t_{M_{k}}^{(k)}, \ldots
$$

which tend to infinity (we set $t_{j}=t_{j}^{(1)}, 1 \leq j \leq M_{1}$ ). Since the local interaction estimate (4.5) holds at these points, the global interaction estimate (5.14) and (5.15) are true for this approximation. Moreover, since the total variation in $x$ of $U^{h}$ is estimated by $L(J)$ together with $\pi(J)$, we have following uniform estimates, provided the initial perturbation is sufficiently small (see Glimm [11], Smoller [22] for the proof).

$$
\begin{array}{ll}
\text { 1. } & T \cdot V \cdot U^{h}(*, t) \leq L_{0}, \\
\text { 2. } & \left\|U^{h}(*, *)\right\|_{L^{\infty}} \leq L_{0}, \\
\text { 3. } & \left\|U^{h}(*, t)-U^{h}(*, s)\right\|_{L^{1}} \leq L_{0}(t-s), \quad t>s
\end{array}
$$

where $L_{0}$ is independent of $h, t$, and $s$. Hence, by Helly's theorem, the approximate solutions $U^{h}$ have a converging subsequence (denoted again by $U^{h}$ ) in $L_{\text {loc }}^{1}$ as $h \rightarrow 0$. Moreover, denoting by $U$ the limit function, we have $F\left(U^{h}\right) \rightarrow F(U)$ in $L_{l o c}^{1}$. Using the truncation estimate (3.3), we can see easily that $U(x, t)$ thus obtained is a weak solution of the conservation laws.

6. Admissibility via Glimm-Lax theory. The Glimm-Lax theory is based on the local interaction estimates (4.5) and the global estimates (5.14). Let us define $\alpha^{+}=\max \{\alpha, 0\}, \alpha^{-}=\min \{0, \alpha\}$ and the cancellation of incoming waves

$$
C_{i}(\mathrm{P})=\frac{1}{2}\left(\sum_{k: i_{k}=i}\left|\alpha_{i_{k}}^{(k)}\right|-\left|\sum_{k: i_{k}=i} \alpha_{i_{k}}^{(k)}\right|\right) .
$$


Then the local interaction estimates (4.5) are expressed as

$$
\epsilon_{i}^{ \pm}=\sum_{k=1}^{p_{i}} \alpha_{i}^{(k) \pm} \mp C_{i}(\mathrm{P})+O(1) Q(\mathrm{P}), \quad i=1,2 .
$$

The notion of generalized characteristic curves was introduced by Glimm and Lax [5]. They defined a broken linear curve $\chi_{i}^{h}(t)(i=1,2)$ issuing from $\left(x_{h}, t_{h}\right)$ in the following way: the curve is either an $i$-characteristic curve or an $i$-shock front passing through $\left(x_{h}, t_{h}\right)$ in the Glimm approximations; when the curve reaches the point of interaction, we extend it as a new $i$-characteristic curve or an $i$-shock front produced by the interaction so that no $i$-rarefaction waves cross it. By the same argument, generalized characteristic curves are defined in the solutions just constructed by the front-tracking alternative, which is briefly accounted in Appendix; in this case $x=\chi_{i}^{h}(t)$ constitutes a Lipschitz continuous curve, which is called an approximate $i$-characteristic curve issuing from $\left(x_{h}, t_{h}\right)$.

Let $\Lambda^{h}$ be a closed region in $R \times R_{+}$surrounded by an approximate $i$-characteristic curve $\chi^{h}$ and segments joining points of interaction. We use the following notation; $L^{ \pm}\left(\Lambda^{h}\right)$ : the total amount of rarefactions $(+)$ and shock waves $(-)$ respectively leaving $\Lambda^{h} ; E^{ \pm}\left(\Lambda^{h}\right)$ : the total amount of rarefactions and shock waves entering $\Lambda^{h}, E^{-}\left(\chi^{h}\right)$ : the total amount of shock waves entering $\chi^{h}$ in $\Lambda^{h} ; X^{ \pm}\left(\chi^{h}\right)$ : the total amount of rarefactions and shock waves crossing $\chi^{h}, C\left(\Lambda^{h}\right)=\sum_{P \in \Lambda^{h}} C(\mathrm{P}), Q\left(\Lambda^{h}\right)=\sum_{P \in \Lambda^{h}} Q(\mathrm{P})$.

LEMMA 6.1 (Approximate conservation laws).

$$
\begin{aligned}
L^{+}\left(\Lambda^{h}\right) & =E^{+}\left(\Lambda^{h}\right)-C\left(\Lambda^{h}\right)+O(1) Q\left(\Lambda^{h}\right), \\
L^{-}\left(\Lambda^{h}\right)+E^{-}\left(\chi^{h}\right) & =E^{-}\left(\Lambda^{h}\right)+C\left(\Lambda^{h}\right)+O(1) Q\left(\Lambda^{h}\right) .
\end{aligned}
$$

The global interaction estimates (5.14)and (6.2) imply

$$
\begin{aligned}
& Q\left(\Lambda^{h}\right) \leq O(1)\left(T . V . U_{0}\right)^{2} \\
& C\left(\Lambda^{h}\right) \leq O(1) T . V . U_{0}+O(1)\left(T . V . U_{0}\right)^{2}
\end{aligned}
$$

and by the above approximate conservation laws

$$
\begin{aligned}
& E^{-}\left(\chi^{h}\right) \leq O(1) \text { T.V. } U_{0}+O(1)\left(\text { T.V. } U_{0}\right)^{2} \\
& X^{ \pm}\left(\chi^{h}\right) \leq O(1) \text { T.V. } U_{0}+O(1)\left(\text { T.V. } U_{0}\right)^{2} .
\end{aligned}
$$

As $h \rightarrow 0,\left(x_{h}, t_{h}\right)$ tends to $\left(x_{0}, t_{0}\right)$ and (a subsequence of) these approximate characteristic curves converges uniformly to a Lipschitz function $\chi_{i}(t)$ on any bounded interval of time; $\chi_{i}(t)$ is called a generalized $i$-characteristic curve issuing from $\left(x_{0}, t_{0}\right)$. Glimm-Lax showed that the derivatives converge pointwisely

$$
\dot{\chi}_{i}(t)=\lim _{h \rightarrow 0} \dot{\chi}_{i}^{h}(t) .
$$

with exception of a certain countable set of values of $t$.

Since the space of bounded Radon measure is compact with respect to the $w^{*}$ topologyCthere exist measures $d Q, d C, d E^{-}(\chi), d X^{ \pm}(\chi)$ such that

$$
\lim _{h \rightarrow 0} Q\left(\Lambda^{h}\right)=\int d Q, \quad \lim _{h \rightarrow 0} C\left(\Lambda^{h}\right)=\int d C,
$$




$$
\lim _{h \rightarrow 0} E^{-}\left(\chi^{h}\right)=\int d E^{-}(\chi), \quad \lim _{h \rightarrow 0} X^{ \pm}\left(\chi^{h}\right)=\int d X^{ \pm}(\chi) .
$$

Let $(\chi(t), t)$ be a point which has measure zero with respect to the measures $(6.4)$ and $t$ has measure zero with respect to (6.5); those $t$ excluded form at most a countable set. At these points, the limit

$$
\lim _{\delta \rightarrow \pm 0} U\left(\chi_{i}(t)+\delta, t\right)=U_{ \pm}(t)
$$

exists and the boundary values $U_{ \pm}(t)$ make sense. Denote $[U(t)]=U_{+}(t)-U_{-}(t)$. If $[U(t)]=0, \chi_{i}(t)$ satisfies the equation of the characteristic curve

$$
\dot{\chi}_{i}(t)=\lambda_{i}\left(U\left(\chi_{i}(t), t\right)\right)
$$

if $[U(t)] \neq 0, \dot{\chi}_{i}(t)$ is the speed of a shock wave

$$
\dot{\chi}_{i}(t)=s_{i}\left(U_{+}(t), U_{-}(t)\right)
$$

which is defined by the Rankine-Hugoniot condition

$$
s[U(t)]=[F(U(t))]
$$

and the Lax entropy condition holds:

$$
\lambda_{i}\left(U_{+}(t)\right)<\dot{\chi}_{i}(t)<\lambda_{i}\left(U_{-}(t)\right)
$$

In order to prove that the solution obtained in the previous section is admissible, we need an analog of (6.6) and (6.8) at the single phase boundary. Like those generalized characteristic curves, a phase boundary issuing from $(0,0)$ is defined in the same way. We show first that the phase boundary thus constructed remains subsonic if the initial perturbation is sufficiently small.

Proposition 6.1. If the total variation of the initial perturbation (1.14) is sufficiently small, then the single phase boundary is subsonic.

Proof. Let $\eta$ denote the quantity (1.14). We observe by Lemma 2.1 that $\dot{\chi}^{h}$ is a function of the states adjoining the phase boundary. Hence by the approximate conservation laws, the total variation of $\dot{\chi}^{h}$ is estimated by that of $U^{h}\left(\chi^{h}(t) \pm 0, t\right)$

$$
\begin{aligned}
\dot{\chi}^{h}(t) & =\dot{\chi}^{h}(0)+O(1) T \cdot V \cdot U^{h}\left(\chi^{h}(t) \pm 0, t\right) \\
& =\dot{\chi}^{h}(0)+O(\eta)+O\left(\eta^{2}\right)
\end{aligned}
$$

which is sufficiently small. Thus the proposition follows.

Proof. [Proof of Theorem 1.3 (admissibility)] Let $\left(\chi\left(t_{0}\right), t_{0}\right)$ be a point on the phase boundary where all the measures (6.4), (6.5) are continuous. There exists a neighborhood $\Delta$ of $\left(\chi\left(t_{0}\right), t_{0}\right)$ and a sequence $\left(\chi^{h}\left(t_{h}\right), t_{h}\right) \in \Delta$ converging to $\left(\chi\left(t_{0}\right), t_{0}\right)$ such that $Q(\Delta), C(\Delta), E\left(\chi^{h} \cap \Delta\right), X^{ \pm}\left(\chi^{h} \cap \Delta\right)$ are arbitrarily small. Since the phase boundary is subsonic and almost stationary, all the waves in $\Delta$ are crossing $\chi^{h}$ and hence the amount of waves are arbitrarily small. Then we find that: for given $\epsilon>0$, there exists $\delta>0$ (depending $\epsilon$ and $t$ ) such that

$$
\left|U^{h}\left(\chi^{h}(t) \pm y, t\right)-U^{h}\left(\chi^{h}(t) \pm 0, t\right)\right|<\epsilon
$$


for $0<y<\delta$. Clearly, the limits (1.15) exist; since the approximate solutions satisfy the kinetic condition (2.7) at the phase boundary, by letting $h \rightarrow 0$, we have (1.16).

The estimate (6.9) is Lemma 3.4 of [12] that is technically the most difficult part of that memoir. The difficulty is to show that: if $\chi_{i}$ is a shock front, every $i$-wave enters $\chi$ with a uniform angle; if $\chi_{i}$ is, on the other hand, a characteristic curve, the amount of $i$-wave in $\Delta$ is uniformly small. Since our phase boundary is subsonic, no such difficulty arises here.

7. Large time stability. In this section, we shall carry out the proof of the stability theorem following the argument in Liu [20] and Asakura [3]. Assume that the initial data satisfies (1.17) and the total variation of the initial perturbation is sufficiently small. Then the characteristic speeds are strictly separated and, by Proposition 6.1 , the phase boundary is subsonic: i.e., there exist constants $\mu_{j}(0 \leq j \leq 3)$ and $\delta>0$ such that

$$
\begin{aligned}
\mu_{0}<\min \left\{\lambda_{1}(U) ; U \in \Omega\right\}-\delta, \quad \max \left\{\lambda_{1}(U) ; U \in \Omega\right\}+\delta<\mu_{1}, \\
\\
\mu_{1}<\dot{\chi}(t)<\mu_{2}, \\
\mu_{2}<\min \left\{\lambda_{2}(U) ; U \in \Omega\right\}-\delta, \quad \max \left\{\lambda_{2}(U) ; U \in \Omega\right\}+\delta<\mu_{3} .
\end{aligned}
$$

Let $\chi_{L}=\left\{\left(x_{L}(t), t\right) ; t \geq 0\right\}$ and $\chi_{R}=\left\{\left(x_{R}(t), t\right) ; t \geq 0\right\}$ be generalized 1 and 2characteristic curves issuing from $(-M, 0)$ and $(M, 0)$, respectively, such that $U(x, t)=$ $U_{m}$ for $x<x_{L}(t)$ and $U(x, t)=U_{m}^{*}$ for $x>x_{R}(t)$. Let $X_{j}^{+}(t)$ and $X_{j}^{-}(t)$ denote respectively the amount of $j$-rarefaction and $j$-shock at time $t$. We also set

$$
X_{j}(t)=X_{j}^{+}(t)+\left|X_{j}^{-}(t)\right|, \quad j=1,2 .
$$

The generalized $j$-characteristic curves through $\left\{\left(x_{L}(t), t\right)\right\}$ and $\left\{\left(x_{R}(t), t\right)\right\}$, respectively, are denoted by $\chi_{j}^{1}(t)$ and $\chi_{j}^{2}(t)$ where $\chi_{j}^{1}$ lies to the left of $\chi_{j}^{2}$. Since the phase boundary is subsonic, there exists $t_{1}>t$ such that $\chi_{2}^{1}(t)$ and $\chi_{1}^{2}(t)$ enter the phase boundary $\chi$ before the time $t_{1}$ and $t_{1}=O(1) t$. For $t \geq t_{1}$, we define $\chi_{1}^{2}(t)$ to be the 1-generalized characteristic curve issuing from $\left(\chi\left(t_{1}\right), t_{1}\right)$ and $\chi_{2}^{1}(t)$ the 2 -generalized characteristic curve issuing from $\left(\chi\left(t_{1}\right), t_{1}\right)$; if these curves start in rarefaction waves, $\chi_{1}^{2}(t)\left(\chi_{2}^{1}(t)\right.$ respectively) is so defined as to run along the right (left, respectively) edge of the rarefaction wave. We set $t_{1}=t_{1}(t)$ and $t^{*}=t_{1}\left(t_{1}(t)\right)$. Clearly there exists a constant $C(C>1)$ depending only on the system and $s_{0}$ such that $t^{*}$ satisfies

$$
t^{*}=C t
$$

For each $t>t_{1}(0), s \geq t_{1}(t)$ and $j=1,2$, we set

$$
\begin{aligned}
\Lambda_{j}(t) & =\text { region between } \chi_{j}^{1}(t) \text { and } c h i_{j}^{2}(t), \\
\Gamma_{+}(t) & =\text { region between } \chi_{1}^{2}(t) \text { and } c h i_{2}^{1}(t) \text { in } x>\chi(t), \\
\Gamma_{-}(t) & =\text { region between } \chi_{1}^{2}(t) \text { and } \chi_{2}^{1}(t) \text { in } x<\chi(t,), \\
D_{j}(s ; t) & =\text { distance between } \chi_{j}^{1}(t) \text { and } \chi_{j}^{2}(t) \text { at time } s, \\
X_{j}^{ \pm}(s ; t) & =\text { amount of } j \text {-waves in the interior of } \Lambda_{j}(t) \text { at time } s, \\
\widetilde{X}_{j}(s ; t) & =\text { amount of } j \text {-waves outside of } \Lambda_{j}(t) \text { at time } s, \\
\operatorname{Str} . \chi_{j}^{i}(s ; t) & =\text { strength of } \chi_{j}^{i}(t) \text { at time } s, \\
Q(t, s) & =\text { amount of interaction between } t<t^{\prime}<s,
\end{aligned}
$$




$$
\begin{gathered}
A(t, s)=\text { amount of approaching waves between } t<t^{\prime}<s, \\
H(t, s)=\text { amount of } i \text {-wave }(i \neq j) \text { crossing } \chi_{j}^{1}(t) \text { and } \chi_{j}^{2}(t) \\
\text { between } t<t^{\prime}<s .
\end{gathered}
$$

We further set

$$
X(t)=\sum_{j=1,2} X_{j}(t), \quad Q(t)=Q(t, \infty)
$$

In the following argument, the bounds $O(1)$ are always independent of $t$ and the solution $U(x, t)$, and only depend on the system.

LEMMA 7.1. There exist bounds $O(1)$ such that for any $s \geq t_{1}(t)$,

$$
\begin{aligned}
\tilde{X}_{j}(s ; t) & =O(1) Q(t) \quad \text { for } j=1,2 \\
H(t, s) & =O(1) Q(t) \\
A\left(s, s^{\prime}\right) & =O(1) Q(t) \quad \text { for } s^{\prime} \geq s \\
\pi\left(s^{\prime}\right)-\pi(s) & =O(1) Q(t) \quad \text { for } s^{\prime} \geq s \\
X\left(t_{1}\right) & \leq O(1) X(t)+O(1) Q(t) \\
Q\left(t_{1}\right) & =O(1) X\left(t_{1}\right)^{3}+O(1) Q(t) X(t)
\end{aligned}
$$

Proof. The approximate conservation laws applied to the region outside of $\Lambda_{j}(t)$ imply (7.2), (7.3) and (7.4). We find by Lemma 4.2, (4.7) that

$$
\left|\pi\left(s^{\prime}\right)-\pi(s)\right| \leq A\left(s, s^{\prime}\right) .
$$

Then (7.5) follows from (7.4). The argument to obtain the global interaction estimates implies

$$
\begin{aligned}
X\left(t_{1}\right) & \leq X(t)+O(1) A\left(t, t_{1}\right)+O(1) Q(t) \\
& \leq\{1+O(1)\} X(t)+O(1) Q(t) .
\end{aligned}
$$

Hence we have (7.6). For (7.7), we observe that the $i$-waves in $\Lambda_{i}(t)$ do not interact with the $j$-waves in $\Lambda_{j}(t)(i \neq j)$. Then it follows from Lemma 5.3 and the definition of $\widetilde{Q}(t)$ that

$$
\begin{aligned}
Q\left(t_{1}\right)=O(1) X\left(t_{1}\right)^{3}+O(1) H\left(t, t_{1}\right) X\left(t_{1}\right)+ & O(1) \sum_{i, j} \widetilde{X}_{i}\left(t_{1}\right) \widetilde{X}_{j}\left(t_{1}\right) \\
& +O(1) Q(t) X\left(t_{1}\right)+O(1) Q(t)^{2}
\end{aligned}
$$

which is $\sum X\left(t_{1}\right)^{3}+O(1) Q(t) X(t)$ by virtue of (7.6), (7.2) and (7.3).

LEMMA 7.2. There exist bounds $O(1)$ such that for any $s \geq t^{*}$ and for any $\left(x_{-}, t_{-}\right) \in \Gamma_{-}(t)$ and any $\left(x_{+}, t_{+}\right) \in \Gamma_{+}(t)$

$$
\begin{aligned}
Q(s) & =O(1) X(t)^{3} \\
\left|U\left(x_{-}, t_{-}\right)-U_{m}\right| & =O(1) X(t)^{3} \\
\left|U\left(x_{+}, t_{+}\right)-U_{m}^{*}\right| & =O(1) X(t)^{3} \\
X_{j}^{+}(s ; t)+X_{j}^{-}(s ; t)+\text { Str. } \chi_{j}^{1}(s ; t) & + \text { Str. } \chi_{j}^{2}(s ; t)
\end{aligned}
$$




$$
=O(1) X(t)^{3} \quad \text { for } j=1,2 \text {. }
$$

Proof. (7.9) is a direct consequence of (7.7) and the definition of $Q(t)$. In order to prove (7.10), (7.11) and (7.12), we need the following lemma (see DiPerna [10]).

LEMMA 7.3. Let $U_{0}, U_{1}, \ldots, U_{n}$ be a sequence of constant states such that $U_{l-1}$ and $U_{l}$ are connected by an arbitrary wave with magnitude $\epsilon_{i}$. Then it follows that, for any $V_{0}$ close to $U_{0}$ and each $j=1,2$, there exists a constant state $V^{(j)}$ lying on the $j$-curve through $V_{0}$ such that

$$
\begin{aligned}
& \left|V_{0}-V^{(j)}\right|=O(1)\left|\sum_{j-\text { waves }} \epsilon\right|+O(1) \sum_{j-\text { shocks }}|\epsilon|^{3}+O(1) \sum_{\text {otherwise }}\left|U_{i}-U_{i-1}\right| \\
& \left|U_{n}-V^{(j)}\right|=O(1)\left|U_{0}-V_{0}\right|+O(1) \sum_{j \text {-shocks }}|\epsilon|^{3}+O(1) \sum_{\text {otherwise }}\left|U_{i}-U_{i-1}\right| .
\end{aligned}
$$

We prove this lemma with the aid of the following propositions (see also [10]).

Proposition 7.1. Let $U_{1}, U_{2}, U_{3}$ be constant states such that $U_{1}$ is arbitrary and that $U_{3}$ is connected to $U_{2}$ by a $j$-wave with magnitude $\epsilon$. Then there exists a constant state $U_{4}$ such that $U_{4}$ is connected to $U_{1}$ by a $j$-wave with magnitude $\epsilon$ and

$$
\left|U_{4}-U_{3}\right|=O(1)\left|U_{1}-U_{2}\right| \text {. }
$$

Proof. Choose $U_{4}$ to be a constant state lying on the $j$-curve through $U_{1}$ with magnitude $\epsilon$. Since the wave curve through $U_{0}$ is differentiable with respect to $U_{0}$, Then (7.15) follows.

Proposition 7.2. Let $U_{1}, U_{2}, U_{3}$ be constant states such that $U_{2}$ is connected to $U_{1}$ by a $j$-wave with magnitude $\epsilon_{1}$ and that $U_{3}$ is connected to $U_{2}$ by a $j$-wave with magnitude $\epsilon_{2}$. Then there exists a constant state $\widetilde{U}_{3}$ lying on the $j$-wave curve through $U_{1}$ such that $\widetilde{U}_{3}$ is a rarefaction wave (or compression wave) with magnitude $\epsilon_{1}+\epsilon_{2}$ and that

$$
\left|U_{3}-\widetilde{U}_{3}\right|=O(1) \sum_{\epsilon_{i}<0}\left|\epsilon_{i}\right|^{3}
$$

Proof. Since $R_{j}\left(U_{0}\right)$ and $T_{j}\left(U_{0}\right)$ contact each other at $U=U_{0}$ with the third order, the deviation of $S_{j}\left(U_{0}\right)$ from $R_{j}\left(U_{0}\right)$ is the third order of the magnitude. Then the proposition follows.

Proof. [Proof of Lemma 7.3] Using Proposition 7.1, we may assume that $U_{i}$ and $U_{i-1}$ are connected by a $j$-wave for $1 \leq i \leq p$ and by other waves for $p<i \leq n$. Obviously

$$
\left|U_{p}-U_{n}\right|=O(1) \sum_{\text {otherwise }}\left|U_{i}-U_{i-1}\right|
$$

Thus we may assume that $n=p$. Now using Proposition 7.2 repeatedly, we can see that there exists a constant state $\widetilde{U}^{(j)}$ lying on the $j$-integral curve through $U_{0}$ with the magnitude

$$
\left|\sum_{i=1}^{n} \epsilon_{i}\right|+O(1) \sum_{\text {shocks }}|\epsilon|^{3}
$$


and

$$
\left|U_{n}-\widetilde{U}^{(j)}\right|=O(1) \sum_{\text {shocks }}|\epsilon|^{3} .
$$

Since the deviation of $S_{j}\left(U_{0}\right)$ from $R_{j}\left(U_{0}\right)$ is the third order of the magnitude, there exists a constant state $U^{(j)}$ on the $j$-wave curve through $U_{0}$ such that

$$
\left|\widetilde{U}^{(j)}-U^{(j)}\right|=O(1) \sum_{\text {shocks }}|\epsilon|^{3}
$$

Then using Proposition 7.1 again, we find a constant state $V^{(j)}$ on the $j$-wave curve through $V_{0}$ such that

$$
\begin{aligned}
\left|V_{0}-V^{(j)}\right| & =O(1)\left|\sum_{i=1}^{n} \epsilon_{i}\right|+O(1) \sum_{j-\text { shocks }}|\epsilon|^{3} \\
\left|U_{n}-V^{(j)}\right| & =O(1)\left|U_{n}-U^{(j)}\right|+O(1)\left|U^{(j)}-V^{(j)}\right| \\
& =O(1) \sum_{j \text {-shocks }}|\epsilon|^{3}+O(1)\left|U_{0}-V_{0}\right|
\end{aligned}
$$

Proof of (7.10)-(7.12). Since $U_{+}(U)$ is differentiable with respect to $U$ by Lemma 2.1 , we have for any $\left(x_{ \pm}, t_{ \pm}\right) \in \Gamma_{ \pm}$

$$
\left|U\left(x_{+}, t_{+}\right)-U_{+}\left(U\left(x_{-}, t_{-}\right)\right)\right|=O(1) Q(t) .
$$

Applying Lemma 7.1 and 7.3 to our approximate solutions, we find that there exist constant states $\widetilde{U}_{m}, \widetilde{U}_{-}, \widetilde{U}_{+}, \widetilde{U}_{m}^{*}$ such that

$$
\widetilde{U}_{m}=U_{m}, \quad \widetilde{U}_{-} \in \mathcal{T}_{1}\left(\widetilde{U}_{m}\right), \quad \tilde{U}_{+}=U_{+}\left(\widetilde{U}_{-}\right), \quad \widetilde{U}_{m}^{*} \in \mathcal{T}_{2}\left(\widetilde{U}_{+}\right)
$$

where $\mathcal{T}_{i}\left(U_{0}\right)$ denotes the $i$-wave curve through $U_{0}$ and

$$
\begin{aligned}
\left|U\left(x_{-}, t_{-}\right)-\widetilde{U}_{-}\right| & =O(1) Q(t)+O(1) \sum_{i=1,2}\left|X_{i}^{-}(t)\right|^{3} \\
\left|U\left(x_{+}, t_{+}\right)-\widetilde{U}_{+}\right| & =O(1) Q(t)+O(1) \sum_{i=1,2}\left|X_{i}^{--}(t)\right|^{3} \\
\left|U_{m}^{*}-\widetilde{U}_{m}^{*}\right| & =O(1) Q(t)+O(1) \sum_{i=1,2}\left|X_{i}^{--}(t)\right|^{3} .
\end{aligned}
$$

Since constant states in the solution to the Riemann problem are differentiable with respect to its initial data, we have

$$
\begin{aligned}
\left|U\left(x_{-}, t_{-}\right)-U_{m}\right| & =O(1) Q(t)+O(1) \sum_{i=1,2}\left|X_{i}^{-}(t)\right|^{3}, \\
\left|U\left(x_{+}, t_{+}\right)-U_{m}^{*}\right| & =O(1) Q(t)+O(1) \sum_{i=1,2}\left|X_{i}^{-}(t)\right|^{3} .
\end{aligned}
$$

By the definition of the magnitude, we can see that for $i=1$,

$$
X_{i}^{+}(s ; t)+X_{i}^{-}(s ; t)+\operatorname{Str} . \chi_{i}^{1}(s ; t)+\operatorname{Str} . \chi_{i}^{2}(s ; t)
$$




$$
\begin{aligned}
& =\lambda_{i}\left(U\left(x_{-}, t_{-}\right)\right)-\lambda_{i}\left(U_{m}\right)+O(1) \tilde{X}_{i}(s ; t) \\
& =O(1)\left|U\left(x_{-}, t_{-}\right)-U_{m}\right|+O(1) Q(t) .
\end{aligned}
$$

We obtain a similar expression for $i=2$. Thus together with (7.9), (7.10) and (7.11) we obtain the lemma.

Proof of Theorem 1.4 (brief outlines). Let us denote

$$
U_{j}^{ \pm i}(t)=U\left(\chi_{j}^{i}(t) \pm 0, t\right), \lambda_{j}^{ \pm i}=\lambda_{j}\left(U_{j}^{ \pm i}(t)\right), \quad i=1,2 .
$$

By (6.8) and (6.7) we have

$$
\frac{d}{d s} D_{j}(s ; t)=s_{j}\left(U_{j}^{+2}(s), U_{j}^{-2}(s)\right)-s_{j}\left(U_{j}^{+1}(s), U_{j}^{-1}(s)\right)
$$

where $s_{j}\left(U_{j}^{+i}(s), U_{j}^{-i}(s)\right)$ denotes $\lambda_{j}\left(U_{j}^{i}(s)\right)$ if $U_{j}^{+i}(s)=U_{j}^{-i}(s)$. Since each characteristic field is genuinely nonlinear, there exists $\theta=\theta(s)(0<\theta<1)$, such that

$$
\begin{aligned}
\frac{d}{d s} D_{j}(s ; t) & =\theta\left[\lambda_{j}^{-2}(s)-\lambda_{j}^{+1}(s)\right]+(1-\theta)\left[\lambda_{j}^{+2}(s)-\lambda_{j}^{-1}(s)\right] \\
& =\left[\lambda_{j}^{-2}(s)-\lambda_{j}^{+1}(s)\right]+(1-\theta)\left[\lambda_{j}^{+2}(s)-\lambda_{j}^{-2}(s)+\lambda_{j}^{+1}(s)-\lambda_{j}^{-1}(s)\right] \\
(7.18) \quad & =X_{j}^{+}(s ; t)+X_{j}^{-}(s ; t)+(1-\theta)\left[\operatorname{Str} \cdot \chi_{j}^{1}(s ; t)+\operatorname{Str} . \chi_{j}^{2}(s ; t)\right]+O(1) \tilde{X}_{j}(s ; t) .
\end{aligned}
$$

Here we use

$$
\lambda_{j}^{-2}(t)-\lambda_{j}^{+1}(t)=X_{j}^{+}(t)+X_{j}^{-}(t)+O(1) \tilde{X}_{j}(t) .
$$

By integrating (7.18) (see [12] for the details), we obtain

$$
X_{j}^{+}(s ; t) \leq \frac{D_{j}(s ; t)}{s-t^{*}}+O(1)|\max \operatorname{Str} .|+O(1) X(t)^{3}
$$

where max Str. denotes the maximum strength of $\chi_{j}^{1}$ and $\chi_{j}^{2}$ between $t^{*}$ and $s$. By applying the argument in [12], section 8 , we can see that above estimate implies the following lemma:

LEMMA 7.4 (Glimm-Lax [12]). For $s \geq t^{*}$, we have

$$
X_{j}^{+}(s ; t) \leq \frac{D_{j}(s ; t)}{s-t^{*}}+O(1) X(t)^{3} .
$$

On the other hand, we recall that

$$
s_{j}\left(U_{j}^{+i}(s), U_{j}^{-i}(s)\right)=\frac{1}{2}\left\{\lambda_{k}\left(U_{j}^{+i}(s)\right)+\lambda_{j}\left(U_{j}^{-i}(s)\right)\right\}+O(1)\left|U_{j}^{+i}(s)-U_{j}^{-i}(s)\right|^{2} .
$$

Hence we have by $(7.17)$

$$
\begin{aligned}
\frac{d}{d s} D_{j}(s ; t)= & \left(\lambda_{j}^{+2}(s)-\lambda_{j}^{-1}(s)\right)-\frac{1}{2}\left\{\lambda_{j}^{+2}(s)-\lambda_{j}^{-2}(s)+\lambda_{j}^{+1}(s)-\lambda_{j}^{-1}(s)\right\} \\
& +O(1)\left|U_{j}^{+1}(s)-U_{j}^{-1}(s)\right|^{2}+O(1)\left|U_{j}^{+2}(s)-U_{j}^{-2}(s)\right|^{2} \\
= & \left(\lambda_{j}^{+2}(s)-\lambda_{j}^{-1}(s)\right)+\left\{\frac{1}{2}+O(1)\left(\operatorname{Str} . \chi_{j}^{1}(s ; t)+\operatorname{Str} . \chi_{j}^{2}(s ; t)\right)\right\}
\end{aligned}
$$




$$
\times\left(\left|\operatorname{Str} . \chi_{j}^{1}(s ; t)\right|+\left|\operatorname{Str} . \chi_{j}^{2}(s ; t)\right|\right) .
$$

Then it follows from Lemmas 7.1 and 7.2 that, for $s \geq t^{*}$

$$
\frac{d}{d s} D_{j}(s ; t)=\left(\lambda_{j}^{+2}(s)-\lambda_{j}^{-1}(s)\right)+\left\{\frac{1}{2}+O(1) X(t)\right\}\left(\mid \text { Str. } \chi_{j}^{1}(s ; t)|+| \text { Str. } \chi_{j}^{2}(s ; t) \mid\right) .
$$

Moreover, we find, by (7.10) and (7.12), that, for $s \geq t^{*}$

$$
\begin{aligned}
\lambda_{j}^{+2}(s)-\lambda_{j}^{-1}(s) & =\left(\lambda_{j}^{+2}(s)-\lambda_{j}\left(U_{m}\right)\right)-\left(\lambda_{j}^{-1}(s)-\lambda_{j}\left(U_{m}\right)\right) \\
& =O(1)\left|U_{j}^{+2}(s)-U_{m}\right|+O(1)\left|U_{j}^{-1}(s)-U_{m}\right| \\
& =O(1) X(t)^{3}, \\
(7.23) \quad \mid \text { Str. } \chi_{j}^{1}(s ; t)|+| \text { Str. } \chi_{j}^{2}(s ; t) \mid & \leq X_{j}^{+}(s ; t)+O(1) X(t)^{3} .
\end{aligned}
$$

Hence, by (7.22) together with (7.24), we have, for $s>t^{*}$

$$
\begin{aligned}
\frac{d}{d s} D_{j}(s ; t) & \leq\left\{\frac{1}{2}+O(1) X(t)\right\} X_{j}^{+}(s ; t)+O(1) X(t)^{3} \\
& \leq\left\{\frac{1}{2}+O(1) X(t)\right\} \frac{D_{j}(s ; t)}{s-t^{*}}+O(1) X(t)^{3} .
\end{aligned}
$$

Using the above differential inequality, we can prove that

$$
X(t)=O(1) t^{-\frac{1}{2}} .
$$

Proof is carried out by integrating (7.25) step by step along the consecutive intervals $[T, C T],\left[C T, C^{2} T\right], \ldots,\left[C^{m} T, C^{m+1} T\right], \ldots$ where $C$ is defined by (7.1) and $T$ large; for the details, we refer the proof of [20], Theorem 7.1, and also [3], Theorem 5.1.

Next we prove

$$
Q(t)=O(1) t^{-\frac{3}{2}}
$$

We observe that the $i$-waves in $\Lambda_{i}(t)$ do not interact with the $j$-waves in $\Lambda_{j}(t)(i \neq j)$ and that the amount of $j$-wave entering $\pi$ is $O(1) Q\left(C^{-1} t\right)$. Hence we see that from definition of $Q(t)$ that

$$
\begin{aligned}
Q(t) & =\max _{i} X_{i}(t) Q\left(C^{-1} t\right)+O(1) Q\left(C^{-1} t\right)^{2}+O(1) \max _{i} X_{i}(t)^{3} \\
& =O(1) t^{-\frac{1}{2}} Q\left(C^{-1} t\right)+O(1) t^{-\frac{3}{2}}=O(1) t^{-\frac{3}{2}}
\end{aligned}
$$

Thus we obtain (7.27). For $\left(x_{ \pm}, t_{ \pm}\right) \in \Gamma_{ \pm}(T)$ for large $T$, we have

$$
\begin{aligned}
\left|U\left(x_{-}, t_{-}\right)-U_{m}\right|,\left|U\left(x_{+}, t_{+}\right)-U_{m}^{*}\right| & =O(1) Q\left(C^{-1} t\right)+O(1) X(t)^{3} \\
& =O(1) t^{-\frac{3}{2}} .
\end{aligned}
$$

In the same manner, we find by (7.10) and (7.11) that

$$
\begin{aligned}
\dot{\chi} & =s(U(\chi-0, t), U(\chi+0, t)) \\
& =s\left(U_{m}, U_{m}^{*}\right)+O(1) Q\left(C^{-1} t\right)+O(1) t^{-\frac{3}{2}}
\end{aligned}
$$




$$
=O(1) t^{-\frac{3}{2}}
$$

Appendix A. Mechanical enthalpy. In this appendix, we shall give the thermodynamic account of the driving traction $f$ given by (1.10). Let us define the mechanical enthalpy function $H$ by

$$
H(s, v)=\frac{1}{2} \widehat{s}^{2}+\int_{0}^{v} \sigma(w) d w-\sigma(v) v
$$

where $\widehat{s}=s v$ : the relative Eulerian shock speed. We have

Proposition A.1. Let $v_{+}, v_{-}$and s satisfy the Rankine-Hugoniot condition (1.6). Then it follows that the driving traction $f$ is expressed as

$$
f\left(v_{+}, v_{-}\right)=H\left(s, v_{+}\right)-H\left(s, v_{-}\right) \text {. }
$$

Proof.

$$
\begin{aligned}
H\left(s, v_{+}\right)-H\left(s, v_{-}\right)= & \frac{1}{2} s^{2}\left(v_{+}^{2}-v_{-}^{2}\right)+\int_{v_{-}}^{v_{+}} \sigma(w) d w-\sigma\left(v_{+}\right) v_{+}+\sigma\left(v_{-}\right) v_{-} \\
= & \frac{1}{2}\left\{\sigma\left(v_{+}\right)-\sigma\left(v_{-}\right)\right\}\left(v_{+}+v_{-}\right)+\int_{v_{-}}^{v_{+}} \sigma(w) d w \\
& \quad-\sigma\left(v_{+}\right) v_{+}+\sigma\left(v_{-}\right) v_{-} \\
= & f\left(v_{+}, v_{-}\right) .
\end{aligned}
$$

Above expression (A.1) will also help us to understand the significance of the kinetic condition (1.12).

Appendix B. Generalized characteristic curves. Let us define, in an approximate solution, a Lipschitz continuous curve $x=\chi^{h}(t)$ issuing from an arbitrary point $\left(x_{0}, t_{0}\right)$ to the $j$-characteristic direction in the following way: if $\left(x_{0}, t_{0}\right)$ is in a constant state, $\chi^{h}$ is the $j$-characteristic curve issuing from this point; if it is a point of interaction, $\chi^{h}$ is one of the $j$-discontinuities; when the $\chi^{h}$ just defined gets to a discontinuity or a point of interaction, we extend this curve according to the following rules.

1. If a $j$-characteristic curve gets to another discontinuity (a shock wave or an approximation of a rarefaction wave) or enters a $j$-shock wave, then the characteristic curve is continued as a $j$-characteristic curve or $j$-shock wave, respectively.

2. If a $j$-discontinuity (a shock wave or an approximation of a rarefaction wave) gets to a point of interaction with discontinuities of another characteristic family, then the $j$-discontinuity is continued as a $j$-rarefaction wave or $j$-shock wave; when more than two $j$-discontinuities are produced as the approximation of the rarefaction wave, we choose the right edge.

3 . If a $j$-discontinuity interacts with other $j$-discontinuities and a $j$-shock wave is produced, then the $j$-discontinuity is continued as a $j$-shock wave producedD

4. If a $j$-discontinuity interacts with other $j$-discontinuities and a $j$-rarefaction wave is produced, the $j$-discontinuity is so defined that the rarefaction wave does not cross the curve. More precisely: 
(a) A left rarefaction wave and a right shock wave interact, then the discontinuity is continued as that of the right edge.

(b) A left shock wave and right rarefaction wave interact, then the discontinuity is continued as the $j$-characteristic curve of the left edge.

We observe that the case $4(\mathrm{~b})$ is not important.

Proposition B.1. If a left shock wave and right rarefaction wave interact, then the amount of rarefaction wave produced by the interaction is $O(1) Q(P)$.

Proof. Assume that the state $U_{L}$ is connected to $U_{M}$ by a $j$-shock wave and the state $U_{M}$ is connected to $U_{R}$ by an approximation of a rarefaction wave. Since two discontinuities interact, it follows that

$$
\lambda_{j}\left(U_{L}\right)>\lambda_{j}\left(U_{R}\right) .
$$

We denote by $U_{j-1}, U_{j}$ the states connected by the $j$-rarefaction wave produced, we have

$$
\lambda_{j}\left(U_{j}\right)-\lambda_{j}\left(U_{j-1}\right)=\lambda_{j}\left(U_{R}\right)-\lambda_{j}\left(U_{L}\right)+O(1) Q(\mathrm{P})<O(1) Q(\mathrm{P}),
$$

because of (B.1). Thus we proved the proposition.

The $j$-approximate characteristic curve $x=\chi^{h}(t)$ issuing from $\left(x_{0}, t_{0}\right)$ is thus constructed.

Acknowledgment. The author would like to thank P. LeFloch, H. Hattori, A. Corli, and M. Tougeron for stimulating discussion and useful instruction. He also thanks referees for helpful suggestion.

\section{REFERENCES}

[1] R. Abeyaratne and J. K. Knowles, On the dissipative response due to discontinuous strains in bars of unstable elastic material, Int. J. Solids and Structures, 24 (1988), pp. 1021-1044.

[2] - Kinetic relations and the propagation of phase boundaries in solids, Arch. Rational Mech. Anal., 114 (1991), pp. 119-154.

[3] F. AsAKURA, Asymptotic stability of solutions with a single strong shock wave for hyperbolic systems of conservation laws, Japan J. Industrial and Applied Math., 11 (1994), pp. 225244.

[4] A. BRESSAN, Global solutions of systems of conservation laws by wave-front tracking, J. Math. Analysis and App., 170 (1992), pp. 414-432.

[5] I.-L. CHERN, Stability theorem and truncation error analysis for the Glimm scheme and for a front tracking method for flows with strong discontinuities, Comm. Pure Appl. Math., 42 (1989), pp. 815-844.

[6] A. CORLI, Non-characteristic phase boundaries for general systems of conservation laws, Preprint.

[7] — Tougeron, Perturbations of bounded variation of a strong shock wave, J. Differential Equations, 138 (1997), pp. 195-228.

[8] _ - Tougeron, Kinetic stabilization of a nonlinear sonic phase boundary, Prépublication 98-21, IRMAR, Univ. Rennes.

[9] C. M. DAFERMOS, The entropy rate admissibility criterion for solutions of hyperbolic conservation laws, J. Differential Equations, 14 (1973), pp. 202-212.

[10] R. J. DIPERNA, Decay of solutions of hyperbolic systems of conservation laws with a convex extensions, Arch. Rat. Mech. Anal., 64(1977), pp. 1-46.

[11] J. GLImM, Solutions in the large for nonlinear hyperbolic systems of equations, Comm. Pure Appl. Math., 18 (1965), pp. 697-715.

[12] J. Glimm and P. D. Lax, Decay of Solutions of Systems of Nonlinear Hyperbolic Conservation Laws, Amer. Math. Soc. Memoir 101. A.M.S., Providence, 1970.

[13] H. HATTORI, The Riemann problem for a van der Waals fluid with entropy rate admissible criterion - Isothermal Case, Arch. Rational Mech. Anal., 92 (1986), pp. 246-263. 
[14] - The Riemann problem and the existence of weak solutions to a system of mixed-type in dynamic phase transition, J. Differential Equations, 146 (1998), pp. 287-319.

[15] R. D. JAMES, The propagation of phase boundaries in elastic bars, Arch. Rational Mech. Anal., 73 (1980), pp. 125-158.

[16] P. D. LAx, Hyperbolic systems of conservation laws II, Comm. Pure Appl. Math., 10 (1957), pp. 537-566.

[17] - Shock waves and entropy, in Contributions to Nonlinear Functional Analysis, E. Zarantonello, ed., Academic Press, New York, 1971, pp.603-634.

[18] P. LeFloch, Propagating Phase boundaries: formulation of the problem and existence via the Glimm method, Arch. Rational Mech. Anal., 123 (1993), pp. 153-197.

[19] T.-P. LIU, Large time behavior of initial and initial-boundary-value problems of general systems of hyperbolic conservation laws, Comm. Math. Phys., 55 (1977), pp. 163-177.

$[20]-$ Decay to $N$-waves of solutions of general systems of nonlinear hyperbolic conservation laws, Comm. Pure Appl. Math., 30 (1977), pp. 585-610.

[21] N. H. Risebro, A front-tracking alternative to the random choice method, Proc. Amer. Math. Soc., 117 (1993), pp. 1125-1139.

[22] J. SmolLer, Shock Waves and Reaction-Diffusion Equations, 2nd ed., Springer-Verlag, New York, 1994. 
